\title{
Efficient asymmetric collisional Brownian particle engines
}

\author{
C. E. Fernández Noa $\odot,{ }^{1}$ Angel L. L. Stable $\odot,{ }^{1}$ William G. C. Oropesa $\odot,{ }^{1}$ Alexandre Rosas,${ }^{2}$ and C. E. Fiore $\odot^{1}$ \\ ${ }^{1}$ Instituto de Física da Universidade de São Paulo, 05508-090 São Paulo, São Paulo, Brazil \\ ${ }^{2}$ Departamento de Física, CCEN, Universidade Federal da Paraíba, Caixa Postal 5008, 58059-900 João Pessoa, Brazil
}

(Received 2 August 2021; accepted 15 November 2021; published 2 December 2021)

\begin{abstract}
The construction of efficient thermal engines operating at finite times constitutes a fundamental and timely topic in nonequilibrium thermodynamics. We introduce a strategy for optimizing the performance of Brownian engines, based on a collisional approach for unequal interaction times between the system and thermal reservoirs. General (and exact) expressions for thermodynamic properties and their optimized values are obtained, irrespective of the driving forces, asymmetry, temperatures of reservoirs, and protocol to be maximized. Distinct routes for the engine optimization, including maximizations of output power and efficiency with respect to the asymmetry, the force, and both of these, are investigated. For the isothermal work-to-work converter and/or a small difference in temperature between reservoirs, they are solely expressed in terms of Onsager coefficients. Although the symmetric engine can operate very inefficiently depending on the control parameters, the usage of distinct contact times between the system and each reservoir not only can enhance the machine performance (signed by an optimal tuning ensuring the largest gain) but also enlarges substantially the machine regime operation. The present approach can pave the way for the construction of efficient Brownian engines operating at finite times.
\end{abstract}

DOI: 10.1103/PhysRevResearch.3.043152

\section{INTRODUCTION}

A long-standing dilemma in thermodynamics and related areas concerns the issue of mitigating the impact of thermal noise or wasted heat in order to improve the machine performance. This constitutes a highly relevant problem, not only for theoretical purposes but also for the construction of experimental setups [1-3]. Giving that the machine performance is commonly dependent on particular chemical compositions and operation conditions, notably for small-scale engines, the role of fluctuations being crucial for such engines, distinct approaches have been proposed and investigated in the realm of stochastic and quantum thermodynamics [4,5]. A second fundamental point concerns the fact that, even if all sources of dissipation could be mitigated, the performance of any thermal machine would still be limited by Carnot efficiency, which requires the occurrence of infinitely slow quasistatic processes, and consequently the engine operates at null power. In contrast, realistic systems operate at finite time and power. Such a conundrum (control or mitigation of dissipation and engine optimization) has contributed to the discovery of several approaches based on the maximization of power output instead of the efficiency [4-20].

Thermal machines based on Brownian particles have been successfully studied not only for theoretical purposes

Published by the American Physical Society under the terms of the Creative Commons Attribution 4.0 International license. Further distribution of this work must maintain attribution to the author $(s)$ and the published article's title, journal citation, and DOI.
$[6,7,15,21]$ but also for the building of reliable experimental setups [22-27]. They are also remarkable for depicting the limitations of classical thermodynamics and disclose the scales at which thermal fluctuations become relevant. In several situations, thermal machines involve isothermal transformations $[22,23,25]$. Such a class of processes are fundamental in thermodynamics since they are minimally dissipative. However, isothermal transformations are slow, demanding a sufficiently large number of stages for achieving the desired final state. For this reason, distinct protocols, such as increasing the coupling between the system and the thermal bath, have been undertaken for speeding it up and simultaneously controlling the increase in dissipation [28-32].

Here, we introduce a strategy for optimizing the performance of irreversible Brownian machines operating in isothermal parts via control of the interaction time between the system and the environment. Our approach is based on a Brownian particle sequentially placed in contact with distinct thermal baths and subject to external forces [33] for unequal times. Such a description, also referred to as collisional, has been successfully employed in different contexts, such as systems that interact only with a small fraction of the environment and those presenting distinct drivings over each member of the system [34-37]. Depending on the parameters of the model (period, driving, and difference of temperatures), the symmetric version can operate very inefficiently. Our aim is to show that the machine performance improves substantially by tuning properly the interaction time between the particle and each reservoir. Besides the increase in the power and/or efficiency, the asymmetry in the contact time also enlarges the regime of operation of the machine substantially. Contrasting with previous works [29-32], the optimization is solely 
obtained via the control of interaction time, and no external parameters are considered. We derive general relations for distinct kinds of maximization, including the maximization of the efficiency and power with respect to the force, the asymmetry, and both of these. For the isothermal work-to-work converter and/or a small difference in temperature between reservoirs, they are solely expressed in terms of Onsager coefficients. The present approach can pave the way for the construction of efficient Brownian engines operating at finite times.

This paper is organized as follows: In Sec. II we present the thermodynamics of Brownian particles subject to asymmetric time switching. In Sec. III, the efficiency is analyzed for two cases: the work-to-work converter processes and distinct temperature reservoirs. Optimization protocols are presented and exemplified for distinct drivings. Finally, conclusions are drawn in Sec. IV, and explicit calculations of the Onsager coefficients and linear regimes are presented in Appendixes A-C.

\section{THERMODYNAMICS OF ASYMMETRIC INTERACTION TIMES}

We consider a Brownian particle with mass $m$ sequentially and cyclically placed in contact with different thermal reservoirs, each at a temperature $T_{i}$ for time interval $\tau_{i}$. Here, $i=1, \ldots, N$ label the reservoirs and also the order of contact between the reservoirs and the particle. While in contact with the $i$ th reservoir, the velocity $v_{i}(t)$ of the particle evolves in time according to the Langevin equation

$$
\frac{d v_{i}}{d t}=-\gamma_{i} v_{i}+f_{i}(t)+\zeta_{i}(t),
$$

where $\gamma_{i}, f_{i}(t)$, and $\zeta_{i}(t)$ denote the viscous constants, external forces, and stochastic forces (interaction between the particle and the $i$ th reservoir), respectively, all divided by the mass of the particle. Stochastic forces are assumed to satisfy the white noise properties

$$
\left\langle\zeta_{i}(t)\right\rangle=0
$$

and

$$
\left\langle\zeta_{i}(t) \zeta_{i^{\prime}}\left(t^{\prime}\right)\right\rangle=2 \gamma_{i} T_{i} \delta_{i i^{\prime}} \delta\left(t-t^{\prime}\right) .
$$

The system evolves to a nonequilibrium steady-state regime (NESS) characterized by a nonvanishing production of entropy. The time evolution of the velocity probability distribution at time $t, P_{i}(v, t)$, is described by the Fokker-Planck equation [38-40]

$$
\frac{\partial P_{i}}{\partial t}=-\frac{\partial J_{i}}{\partial v}-f_{i}(t) \frac{\partial P_{i}}{\partial v},
$$

where $J_{i}$ is the probability current

$$
J_{i}=-\gamma_{i} v P_{i}-\frac{\gamma_{i} k_{\mathrm{B}} T_{i}}{m} \frac{\partial P_{i}}{\partial v} .
$$

As can be verified by direct substitution, the NESS is characterized by a Gaussian probability distribution $P_{i}(v, t)$ :

$$
P_{i}(v, t)=\frac{1}{\sqrt{2 \pi b_{i}(t)}} e^{-\frac{\left(v-\left\langle v_{i}\right\rangle\right)^{2}}{2 b_{i}(t)}},
$$

for which the mean $\left\langle v_{i}\right\rangle(t)$ and the variance $b_{i}(t) \equiv\left\langle v_{i}^{2}\right\rangle(t)-$ $\left\langle v_{i}\right\rangle^{2}(t)$ are time dependent and obey the following equations of motion:

$$
\frac{d}{d t}\left\langle v_{i}\right\rangle(t)=-\gamma_{i}\left\langle v_{i}\right\rangle(t)+f_{i}(t)
$$

and

$$
\frac{d}{d t} b_{i}(t)=-2 \gamma_{i} b_{i}(t)+\Gamma_{i},
$$

where $\Gamma_{i}=2 \gamma_{i} k_{\mathrm{B}} T_{i} / m$. Obviously, the continuity of the probability distribution must be assured, and we will use it to calculate $b_{i}(t)$ and $\left\langle v_{i}\right\rangle(t)$ in the following sections.

In order to derive explicit expressions for macroscopic quantities, we start from the definitions of the average energy $U_{i}=m\left\langle v_{i}^{2}\right\rangle / 2$ and entropy $S_{i}(t)=-k_{\mathrm{B}}\left\langle\ln \left[P_{i}(v, t)\right]\right\rangle$, respectively. In both cases, the time variation can be straightforwardly obtained from the Fokker-Planck equation, applying vanishing boundary conditions for both $P_{i}(v, t)$ and $J_{i}(v, t)$ in the infinity-speed limit [38]. The former is related to the average power dissipated $\dot{W}_{i}$ and the heat dissipation during the same period $\dot{Q}_{i}$ through the first law of thermodynamics relation

$$
\frac{d U_{i}}{d t}=-\left[\dot{W}_{i}(t)+\dot{Q}_{i}(t)\right]
$$

where $\dot{W}_{i}(t)$ and $\dot{Q}_{i}(t)$ are given by the following expressions:

$$
\dot{W}_{i}(t)=-m\left\langle v_{i}\right\rangle(t) f_{i}(t)
$$

and

$$
\dot{Q}_{i}(t)=m \gamma_{i}\left(\left\langle v_{i}^{2}\right\rangle(t)-\frac{\Gamma_{i}}{2 \gamma_{i}}\right) .
$$

Similarly, the rate of variation of the entropy can be written as $[39,40]$

$$
\frac{d S_{i}}{d t}=\Pi_{i}(t)-\Phi_{i}(t),
$$

where $\Pi_{i}(t)$ and $\Phi_{i}(t)$ denote the entropy production rate and the flux of entropy, respectively, which expressions are given by

$$
\Pi_{i}(t)=\frac{2 k_{\mathrm{B}}}{\Gamma_{i}} \int_{\tilde{\tau}_{i-1}}^{t} \frac{J_{i}^{2}}{P_{i}} d v
$$

and

$$
\Phi_{i}(t)=-\frac{2 \gamma_{i} k_{\mathrm{B}}}{\Gamma_{i}} \int_{\tilde{\tau}_{i-1}}^{t} v J_{i} d v=\frac{2 \gamma_{i} k_{\mathrm{B}} \dot{Q}_{i}(t)}{m \Gamma_{i}}=\frac{\dot{Q}_{i}(t)}{T_{i}},
$$

respectively, where $\tilde{\tau}_{i}=\sum_{j=1}^{i} \tau_{j}$ (with $\tau_{0} \equiv 0$ ). Both expressions are valid during the contact of the Brownian particle with the $i$ th reservoir.

As stated before, the present collisional approach for Brownian machines can be considered for an arbitrary set of reservoirs and external forces, whose generic solutions $\left\langle v_{i}\right\rangle(t)$ and $b_{i}(t)$ in the nonequilibrium steady-state regime are

$$
\left\langle v_{i}\right\rangle(t)=e^{-\gamma_{i}\left(t-\tilde{\tau}_{i-1}\right)} a_{i}+e^{-\gamma_{i} t} F_{i}(t)
$$

and

$$
b_{i}(t)=A_{i} e^{-2 \gamma_{i}\left(t-\tilde{\tau}_{i-1}\right)}+\frac{\Gamma_{i}}{2 \gamma_{i}},
$$


where $a_{i}$ and $A_{i}$ are integration constants to be determined from the boundary conditions and $F_{i}(t)$ can be viewed as a "time-integrated force," which is related to the external forces through the expression

$$
F_{i}(t)=\int_{\tilde{\tau}_{i-1}}^{t} e^{\gamma_{i} t^{\prime}} f_{i}\left(t^{\prime}\right) d t^{\prime} .
$$

Here, the variable $t$ is interpreted as the time modulus the period $\tau=\tilde{\tau}_{N}$.

Since the probability distribution is continuous, the conditions $\left\langle v_{i}\right\rangle\left(\tau_{i}\right)=\left\langle v_{i+1}\right\rangle\left(\tau_{i}\right)$ and $b_{i}\left(\tau_{i}\right)=b_{i+1}\left(\tau_{i}\right)$ must hold for $i=1, \ldots, N-1$. In addition, the steady-state condition (periodicity) implies that $\left\langle v_{1}\right\rangle(0)=\left\langle v_{N}\right\rangle(\tau)$ and $b_{1}(0)=b_{N}(\tau)$. Hence the $a_{i}$ and $A_{i}$ can be determined as the solution of two uncoupled linear systems of $N$ equations each. Here, we shall focus on the case of $N=2$ reservoirs-the simplest case for tackling the efficiency of a thermal engine, in which the interaction with the first and second reservoirs occurs during $\tau_{1}$ and $\tau_{2}=\tau-\tau_{1}$, respectively. For simplicity, from now on, we consider that the viscous constants are equal $\gamma_{1}=\gamma_{2}=\gamma$. Therefore the average velocities and their variances are

$$
\begin{aligned}
&\left\langle v_{1}\right\rangle(t)=\frac{\left(e^{\gamma \tau}-1\right) F_{1}(t)+F_{1}\left(\tau_{1}\right)+F_{2}(\tau)}{e^{\gamma t}\left(e^{\gamma \tau}-1\right)}, \quad(18) \begin{array}{l}
\text { Using Eq. (18) and expressing each external force } \\
X_{i} g_{i}(t), \text { with } X_{i} \text { and } g_{i}(t) \text { denoting force strength } \\
\text { driving, respectively, we finally arrive at the following } \\
\text { sions: }
\end{array} \\
&\left\langle v_{2}\right\rangle(t)=\frac{e^{\gamma \tau} F_{1}\left(\tau_{1}\right)+\left(e^{\gamma \tau}-1\right) F_{2}(t)+F_{2}(\tau)}{e^{\gamma t}\left(e^{\gamma \tau}-1\right)}, \\
& \overline{\dot{W}}_{1}=-\frac{m}{\tau\left(e^{\gamma \tau}-1\right)}\left[X_{1}^{2}\left(\left(e^{\gamma \tau}-1\right) \int_{0}^{\tau_{1}} g_{1}(t) e^{-\gamma t} d t \int_{0}^{t} g_{1}\left(t^{\prime}\right) e^{\gamma t^{\prime}} d t^{\prime}+\int_{0}^{\tau_{1}} g_{1}(t) e^{-\gamma t} d t \int_{0}^{\tau_{1}} g_{1}\left(t^{\prime}\right) e^{\gamma t^{\prime}} d t^{\prime}\right)\right.
\end{aligned}
$$

The expressions above, Eqs. (22) and (23), are exact and are valid for any kind of drivings $g_{1}(t)$ and $g_{2}(t)$ and stage duration $\tau_{1}$ and $\tau_{2}$. Usually, in the linear regime, $\dot{W}_{i}$ is written as the product of a flux $\mathcal{J}_{i}=L_{i i} X_{i}+L_{i j} X_{j}$ by a force $X_{i}$; that is, $\overline{\dot{W}}_{i}=-k_{\mathrm{B}} T_{i} \mathcal{J}_{i} X_{i}$. Since in the present case $\overline{\dot{W}}_{i}$ is always bilinear in the forces $X_{i}$, such an expression is also valid even far from the linear regime. Thus the Onsager coefficients $L_{i j}$ may be written as

$$
\begin{aligned}
& L_{11}=\frac{2 \gamma}{\Gamma_{1} \tau\left(e^{\gamma \tau}-1\right)}\left[\left(e^{\gamma \tau}-1\right) \int_{0}^{\tau_{1}} g_{1}(t) e^{-\gamma t} d t \int_{0}^{t} g_{1}\left(t^{\prime}\right) e^{\gamma t^{\prime}} d t^{\prime}+\int_{0}^{\tau_{1}} g_{1}(t) e^{-\gamma t} d t \int_{0}^{\tau_{1}} g_{1}\left(t^{\prime}\right) e^{\gamma t^{\prime}} d t^{\prime}\right], \\
& L_{22}=\frac{2 \gamma}{\Gamma_{2} \tau\left(e^{\gamma \tau}-1\right)}\left[\int_{\tau_{1}}^{\tau} g_{2}(t) e^{-\gamma t} d t \int_{\tau_{1}}^{\tau} g_{2}\left(t^{\prime}\right) e^{\gamma t^{\prime}} d t^{\prime}+\left(e^{\gamma \tau}-1\right) \int_{\tau_{1}}^{\tau} g_{2}(t) e^{-\gamma t} d t \int_{\tau_{1}}^{t} g_{2}\left(t^{\prime}\right) e^{\gamma t^{\prime}} d t^{\prime}\right], \\
& L_{12}=\frac{2 \gamma}{\Gamma_{1} \tau\left(e^{\gamma \tau}-1\right)} \int_{0}^{\tau_{1}} g_{1}(t) e^{-\gamma t} d t \int_{\tau_{1}}^{\tau} g_{2}\left(t^{\prime}\right) e^{\gamma t^{\prime}} d t^{\prime}, \\
& L_{21}=\frac{2 \gamma e^{\gamma \tau}}{\Gamma_{2} \tau\left(e^{\gamma \tau}-1\right)} \int_{0}^{\tau_{1}} g_{1}\left(t^{\prime}\right) e^{\gamma t^{\prime}} d t^{\prime} \int_{\tau_{1}}^{\tau} g_{2}(t) e^{-\gamma t} d t .
\end{aligned}
$$


Reciprocal relations are verified as follows: Since forces $f_{1}(t)$ and $f_{2}(t)$ solely act from 0 to $\tau_{1}$ and $\tau_{1}$ to $\tau$, respectively, both upper and lower integral limits in Eqs. (20) and (21) can be replaced for $\tau$ and 0 , respectively, and hence all expressions from Eq. (20) to Eq. (24) can be evaluated over a complete cycle. By exchanging the indexes $1 \leftrightarrow 2$, we verify that $L_{i j} \leftrightarrow L_{j i}$.

Similarly, general expressions can be obtained for the average heat dissipation during the contact of the Brownian particle with each reservoir. Since the heat is closely related to the entropy production rate [see, e.g., Eq. (14)], we curb our discussion to the latter quantity. The average entropy production over a complete cycle is then given by

$$
\bar{\Pi}=\frac{1}{\tau}\left[\int_{0}^{\tau_{1}} \Phi_{1}(t) d t+\int_{\tau_{1}}^{\tau} \Phi_{2}(t) d t\right] .
$$

By inserting Eq. (14) into Eq. (25) and using Eq. (11), $\bar{\Pi}$ can be decomposed into two terms: one associated with the difference in temperature of the reservoirs

$$
\bar{\Pi}_{T}=\frac{k_{\mathrm{B}}}{\tau}\left[\frac{2 \gamma^{2}}{\Gamma_{1}} \int_{0}^{\tau_{1}} b_{1}(t) d t+\frac{2 \gamma^{2}}{\Gamma_{2}} \int_{\tau_{1}}^{\tau} b_{2}(t) d t-\gamma \tau\right],
$$

and the other coming from the external forces

$$
\bar{\Pi}_{F}=\frac{k_{\mathrm{B}}}{\tau}\left[\frac{2 \gamma^{2}}{\Gamma_{1}} \int_{0}^{\tau_{1}}\left\langle v_{1}\right\rangle^{2}(t) d t+\frac{2 \gamma^{2}}{\Gamma_{2}} \int_{\tau_{1}}^{\tau}\left\langle v_{2}\right\rangle^{2}(t) d t\right] .
$$

Now, from Eqs. (19) and (26), one obtains the general form for $\bar{\Pi}_{T}$ :

$$
\bar{\Pi}_{T}=k_{\mathrm{B}} \frac{\Gamma_{1} \Gamma_{2}}{\tau} \frac{\sinh \left(\gamma \tau_{1}\right) \sinh \left(\gamma \tau_{2}\right)}{\sinh (\gamma \tau)}\left(\frac{1}{\Gamma_{1}}-\frac{1}{\Gamma_{2}}\right)^{2},
$$

which is strictly positive (as expected). The component $\left(1 / \Gamma_{1}-1 / \Gamma_{2}\right)$ can be regarded as the "thermodynamic force" $f_{\Gamma}$ associated with the difference in temperature of the reservoirs. Particularly, in the linear regime $\left(\Gamma_{2} \simeq \Gamma_{1}=\Gamma\right), \bar{\Pi}_{T}$ can be conveniently written down in terms of Onsager coefficient $\bar{\Pi}_{T}=L_{\Gamma \Gamma} f_{\Gamma}$, where $L_{\Gamma \Gamma}$ is given by

$$
L_{\Gamma \Gamma}=k_{\mathrm{B}} \frac{\Gamma^{2}}{\tau} \frac{\sinh \left(\gamma \tau_{1}\right) \sinh \left(\gamma \tau_{2}\right)}{\sinh (\gamma \tau)} .
$$

Note that $L_{\Gamma \Gamma}$ is strictly positive and it reduces to $k_{\mathrm{B}} \Gamma^{2} \tanh \left[\frac{\gamma \tau}{2}\right] / 2 \tau$ for $\tau_{1}=\tau_{2}$ (symmetric case). Furthermore, it is straightforward to verify that the dissipation term $\Pi_{T}$ is a monotonous decreasing function of $\tau$ and it is always larger for the symmetric case $\left(\tau_{1}=\tau_{2}\right)$. Both properties of $\bar{\Pi}_{T}$ are illustrated in Fig. 1, where $\bar{\Pi}_{T}$ is shown as a function of $\tau$ for various values of the asymmetry parameter $\kappa=\tau_{1} / \tau_{2}$ (notice that $\bar{\Pi}_{T}$ is invariant over the switch of the interaction times $\tau_{1} \leftrightarrow \tau_{2}$ or, equivalently, $\left.\kappa \leftrightarrow 1 / \kappa\right)$. There is one caveat which concerns the validity of the results of Fig. 1. Collisional models usually neglect the time for changing the contact between the system and thermal baths. However, if $\tau$ is very small, such an approximation can no longer hold. We shall assume throughout this paper that $\tau$ is large enough for the collisional approximation to be valid.

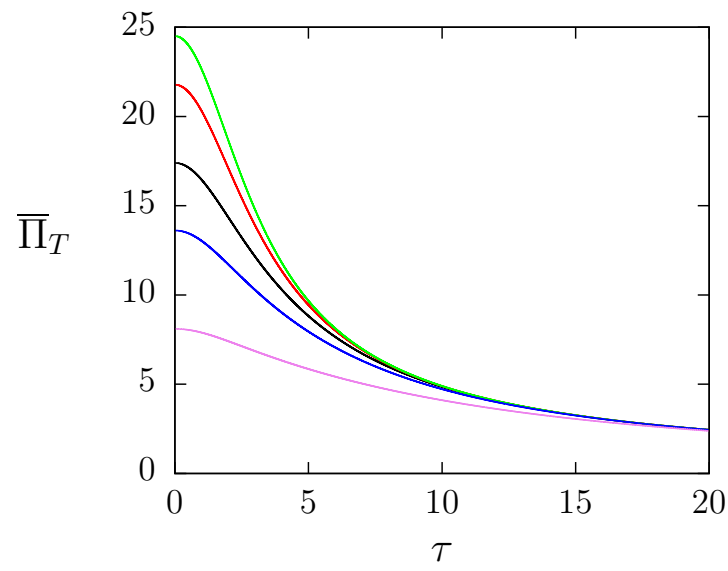

FIG. 1. Mean entropy production component $\bar{\Pi}_{T}$ as a function of the period $\tau$ for $\gamma=1, \Gamma_{1}=1$, and $\Gamma_{2}=100$ and distinct asymmetries. From top to bottom: $\kappa=1.0,0.5,0.3,0.2$, and 0.1 .

The entropy production component coming from external forces also assumes a general (bilinear) form given by

$$
\bar{\Pi}_{F}=\tilde{L}_{11} X_{1}^{2}+\left(\tilde{L}_{12}+\tilde{L}_{21}\right) X_{1} X_{2}+\tilde{L}_{22} X_{2}^{2} .
$$

The coefficients $\tilde{L}_{i j}$ are shown in Appendix A, Eq. (A2). It should be noticed that Eq. (30) is exact for all force regimes (not only in the linear regime). For equal temperatures, they coincide with the Onsager coefficients [Eq. (24)]. A detailed analysis for distinct linear regimes (low temperature difference and/or low forces) is undertaken in Appendix A. Furthermore, since $\tau_{2}=\tau-\tau_{1}$, the coefficients above fulfill the reciprocal relations $\tilde{L}_{11} \leftrightarrow \tilde{L}_{22}$ and $\tilde{L}_{12} \leftrightarrow \tilde{L}_{21}$ by exchanging $1 \leftrightarrow 2$ for the generic drivings $g_{i}(t)$, the interaction times $\tau_{i}$, and the temperature of the reservoirs $T_{i}$.

\section{EFFICIENCY}

The optimization of engines, which converts energy (usually heat or chemical work) into mechanical work, constitutes one of the main issues in thermodynamics, engineering, chemistry, and other fields. Here, we exploit the role of asymmetric contact times between the Brownian particle and the thermal reservoirs as a reliable strategy for optimizing the machine performance. More specifically, the amount of energy (heat and work) received by the particle is partially converted into output work (or, equivalently, the output power per cycle) $\mathcal{P}=$ $\dot{W}_{2} \geqslant 0$ during the second half stage. A measure of efficiency is given by the ratio of the amount of output work to the total energy injected:

$$
\eta=-\frac{\mathcal{P}}{\overline{\dot{W}_{1}}+\overline{\dot{Q}}_{i}}
$$

where $\overline{Q_{i}}$ is the average heat extracted from the reservoir $i$ ( $i=1$ or 2 depending on whether reservoir 1 or 2 delivers heat to the Brownian particle), whereas for the other way round (both reservoirs absorbing energy from the particle), $\overline{\dot{Q}}_{i}$ does not appear in Eq. (31), as shall be discussed in Sec. III A. It is worth mentioning that in the case of more than two reservoirs, the numerator of the efficiency should be the total 
power extracted from the systems (sum of all $\bar{W}_{i}>0$ ) and the denominator is the total power injected into the system (sum of all ${\overline{W_{i}}}_{i}<0$ ) plus the total heat injected into the system (sum of all $\overline{\dot{Q}_{i}}<0$ ).

Below, we are going to investigate the machine optimization with respect to the loading force $X_{2}$ and asymmetry coefficient $\kappa=\tau_{1} / \tau_{2}$ for two distinct scenarios: equal and different temperatures.

\section{A. Isothermal work-to-work converter}

Many processes in nature, such as biological systems, operate at homogeneous (or approximately equal) temperatures, in which an amount of chemical work or energy is converted into mechanical work and vice versa (see, e.g., Refs. [41,42]). This highlights the importance of searching for optimized protocols operating at equal temperatures. Here, we exploit the present Brownian machine operating at equal temperatures, but subject to distinct external forces. From Eqs. (11) and (19), it follows that $\overline{\dot{Q}_{1}} \geqslant 0$ and $\overline{\dot{Q}_{2}} \geqslant 0$, and therefore no heat is delivered to the particle. Such an engine reduces to a workto-work converter: The particle receives input power $\overline{\dot{W}_{1}}<0$ which is partially converted into output power $\mathcal{P} \geqslant 0$. From Eq. (24), the output power and efficiency can be expressed in terms of the Onsager coefficients according to the following expressions:

$$
\mathcal{P}=\overline{\dot{W}}_{2}=-k_{\mathrm{B}} T\left[L_{22}(\kappa) X_{2}^{2}+L_{21}(\kappa) X_{1} X_{2}\right]
$$

and

$$
\eta=-\frac{L_{21} X_{1} X_{2}+L_{22} X_{2}^{2}}{L_{11} X_{1}^{2}+L_{12} X_{1} X_{2}}
$$

Both of them can be expressed in terms of the ratio $X_{2} / X_{1}$ between forces, the output power being a function of such a ratio multiplied by $X_{1}^{2}$. As mentioned previously, there are three routes to be considered with respect to the engine optimization (holding $X_{1}$ and $\tau$ fixed): the time asymmetry optimization (conveniently carried out in terms of the ratio $\kappa=\tau_{1} / \tau_{2}$ ), the output force $X_{2}$ optimization, and both optimizations together. We shall analyze all cases in Secs. III A 1 to III A 4.

\section{Maximization with respect to the asymmetry}

Since the Brownian particle must be in contact with the first reservoir long enough for the injected energy to be larger than the energy dissipated by the viscous force, for any set of $X_{1}$ and $X_{2}$ there is a minimum value $\kappa_{m}$ for which $\mathcal{P} \geqslant 0$. On the other hand, depending on the kind of driving, it can extend up $\kappa \rightarrow \infty$, for which $L_{21}$ and $L_{22}$ vanish [see Eq. (24)].

The choice of optimal asymmetries is expected to be dependent on the quantity chosen to be maximized. Usually, there are two quantities of interest: maximum efficiency or maximum power output. Starting with the latter case, the optimal asymmetry $\kappa_{M P}$ which maximizes $\mathcal{P}$ is the solution of the following equation:

$$
\frac{L_{21}^{\prime}\left(\kappa_{M P}\right)}{L_{22}^{\prime}\left(\kappa_{M P}\right)}=-\frac{X_{2}}{X_{1}},
$$

where $L_{i j}^{\prime}(\kappa) \equiv \partial L_{i j}(\kappa) / \partial \kappa$. In this section, $L_{i j}$ 's (together with their derivatives) have been expressed in terms of $\kappa$ to specify which quantity ( $\mathcal{P}$ or $\eta$ ) has been maximized. In general, Eq. (34) may have more than one solution for each choice of the ratio $X_{2} / X_{1}$, and one should be careful to identify the global maximum. However, in the following discussion (as in the examples presented in Sec. III A 3), we consider the cases which present a single maximum.

Similarly, from Eq. (33), we obtain the value of the asymmetry that maximizes the efficiency $\kappa_{M \eta}$ from the transcendental equation

$$
\begin{aligned}
\Delta_{2212}\left(\kappa_{M \eta}\right) & X_{2}^{2}+\Delta_{2111}\left(\kappa_{M \eta}\right) X_{1}^{2} \\
& +\left[\Delta_{2211}\left(\kappa_{M \eta}\right)+\Delta_{2112}\left(\kappa_{M \eta}\right)\right] X_{1} X_{2}=0,
\end{aligned}
$$

where $\Delta_{i j k l}(\kappa)=L_{i j}^{\prime}(\kappa) L_{k l}(\kappa)-L_{k l}^{\prime}(\kappa) L_{i j}(\kappa)$. Although exact, for a given choice of the drivings $g_{i}(t)$ and the strengths $X_{i}$, Eqs. (34) and (35), in general, have to be solved numerically for $\kappa_{M P}$ and $\kappa_{M \eta}$, respectively. After these values are obtained, we can evaluate the power $\mathcal{P}_{M P, \kappa}$ and efficiency $\eta_{M P, \kappa}$ at maximum power as

$$
\begin{aligned}
\mathcal{P}_{M P, \kappa}= & \frac{k_{\mathrm{B}} T L_{21}^{\prime}\left(\kappa_{M P}\right)}{L_{22}^{\prime 2}\left(\kappa_{M P}\right)}\left[L_{21}\left(\kappa_{M P}\right) L_{22}^{\prime}\left(\kappa_{M P}\right)\right. \\
& \left.-L_{22}\left(\kappa_{M P}\right) L_{21}^{\prime}\left(\kappa_{M P}\right)\right] X_{1}^{2}
\end{aligned}
$$

and

$\eta_{M P, \kappa}=\frac{L_{21}^{\prime}\left(\kappa_{M P}\right)\left[L_{22}^{\prime}\left(\kappa_{M P}\right) L_{21}\left(\kappa_{M P}\right)-L_{22}\left(\kappa_{M P}\right) L_{21}^{\prime}\left(\kappa_{M P}\right)\right]}{L_{22}^{\prime}\left(\kappa_{M P}\right)\left[L_{11}\left(\kappa_{M P}\right) L_{22}^{\prime}\left(\kappa_{M P}\right)-L_{12}\left(\kappa_{M P}\right) L_{21}^{\prime}\left(\kappa_{M P}\right)\right]}$.

Analogously, we can write the power at maximum efficiency $\mathcal{P}_{M \eta, \kappa}$ and maximum efficiency $\eta_{M \eta, \kappa}$ as

$$
\mathcal{P}_{M \eta, \kappa}=-k_{\mathrm{B}} T\left[L_{22}\left(\kappa_{M \eta}\right) X_{2}^{2}+L_{21}\left(\kappa_{M \eta}\right) X_{1} X_{2}\right]
$$

and

$$
\eta_{M \eta, \kappa}=-\frac{L_{22}\left(\kappa_{M \eta}\right) X_{2}^{2}+L_{21}\left(\kappa_{M \eta}\right) X_{1} X_{2}}{L_{11}\left(\kappa_{M \eta}\right) X_{1}^{2}+L_{12}\left(\kappa_{M \eta}\right) X_{1} X_{2}}
$$

respectively. In Sec. III A 3, we will exemplify our exact expressions for maximum efficiencies and powers for two kinds of drivings.

\section{Maximization with respect to the output force}

For given asymmetry and drivings, the Onsager coefficients are constant. Hence the maximization of the output power and the efficiency turns out to be similar to the approach from Refs. [16,33]. Below, we recast the main results.

As previously, the engine regime $(\mathcal{P}>0)$ also imposes boundaries to optimization with respect to the force strength. Here, the output force $X_{2}$ must lie in the interval $X_{m} \leqslant X_{2} \leqslant 0$, where $X_{m}=-L_{21} X_{1} / L_{22}$. In general, $X_{m}$ is different from the value of the output force that minimizes the entropy production $X_{2 m s}$ (for $X_{1}$ and $\kappa$ constants). According to Eq. (30), such a difference is given by $X_{2 m}-X_{2 m S}=\left(L_{12}-L_{21}\right) X_{1} / 2 L_{22}$. Note that they coincide, $X_{m}=X_{2 m s}$, for symmetric Onsager coefficients $L_{12}=L_{21}$, but they are different when $L_{12} \neq L_{21}$. Similarly to Sec. III A 1, the optimization can be performed to ensure maximum power $\mathcal{P}_{M P, X_{2}}$ (with efficiency $\eta_{M P, X_{2}}$ ) or maximum efficiency $\eta_{M \eta, X_{2}}$ (with power $\mathcal{P}_{M \eta, X_{2}}$ ), by adjusting 
the output forces to optimal values $X_{2 M P}$ and $X_{2 M \eta}$, respectively. These optimal output forces can be expressed in terms of the Onsager coefficients as

$$
X_{2 M \eta}=\frac{L_{11}}{L_{12}}\left(-1+\sqrt{1-\frac{L_{12} L_{21}}{L_{11} L_{22}}}\right) X_{1}
$$

and

$$
X_{2 M P}=-\frac{1}{2} \frac{L_{21}}{L_{22}} X_{1},
$$

respectively. Hence the maximum efficiency $\eta_{M \eta, X_{2}}$ and the efficiency at maximum power $\eta_{M P, X_{2}}$ are given by

$$
\eta_{M \eta, X_{2}}=-\frac{L_{21}}{L_{12}}+\frac{2 L_{11} L_{22}}{L_{12}^{2}}\left(1-\sqrt{1-\frac{L_{12} L_{21}}{L_{11} L_{22}}}\right)
$$

and

$$
\eta_{M P, X_{2}}=\frac{L_{21}^{2}}{4 L_{11} L_{22}-2 L_{12} L_{21}},
$$

while the power at maximum efficiency $\mathcal{P}_{M \eta, X_{2}}$ and the maximum power $\mathcal{P}_{M P, X_{2}}$ can obtained by inserting $X_{2 M \eta}$ or $X_{2 M P}$ into the expression for $\mathcal{P}$. In fact, these quantities are not independent of each other; instead they are related as

$$
\eta_{M P, X_{2}}=\frac{P_{M P, X_{2}}}{2 P_{M P, X_{2}}-P_{M \eta, X_{2}}} \eta_{M \eta, X_{2}} .
$$

Furthermore, for symmetric Onsager coefficients $L_{12}=L_{21}$, there are two additional simple relations given by

$$
\eta_{M P, X_{2}}=\frac{\eta_{M \eta, X_{2}}}{1+\eta_{M \eta, X_{2}}^{2}} \quad \text { and } \quad \frac{\mathcal{P}_{M \eta, X_{2}}}{\mathcal{P}_{M P, X_{2}}}=1-\eta_{M \eta, X_{2}}^{2} .
$$

As shown in Appendix B, $L_{12}=L_{21}$ for constant drivings for any value of $\kappa$. Conversely, they are in general different $\left(L_{12} \neq\right.$ $L_{21}$ ) for linear drivings (see Appendix C). For the symmetric time case $(\kappa=1)$, however, the equality holds also for linear drivings [33].

\section{Constant and linear drivings}

In order to access the advantages of the asymmetry in the time spent by the Brownian particle in contact with each reservoir, we consider two different driving models. In the first model, the drivings are constant, and the external forces can be written as

$$
\begin{aligned}
& f_{1}(t)=X_{1}, \quad \text { for } 0 \leqslant t<\tau_{1}, \\
& f_{2}(t)=X_{2}, \quad \text { for } \tau_{1} \leqslant t<\tau .
\end{aligned}
$$

In Appendix B, we present explicit expressions for the average velocities $\left\langle v_{i}\right\rangle(t)$ and Onsager coefficients $L_{i j}$ (which coincide with the coefficients $\tilde{L}_{i j}$ for isothermal reservoirs). The second class of Brownian engines deals with drivings evolving linearly in time and given by the following expressions:

$$
\begin{gathered}
f_{1}(t)=X_{1} \gamma t, \quad \text { for } 0 \leqslant t<\tau_{1}, \\
f_{2}(t)=X_{2} \gamma\left(t-\tau_{1}\right), \quad \text { for } \tau_{1} \leqslant t<\tau .
\end{gathered}
$$

The main expressions for such a case are listed in Appendix C. Figures 2 and 3 depict typical plots of the efficiency and power
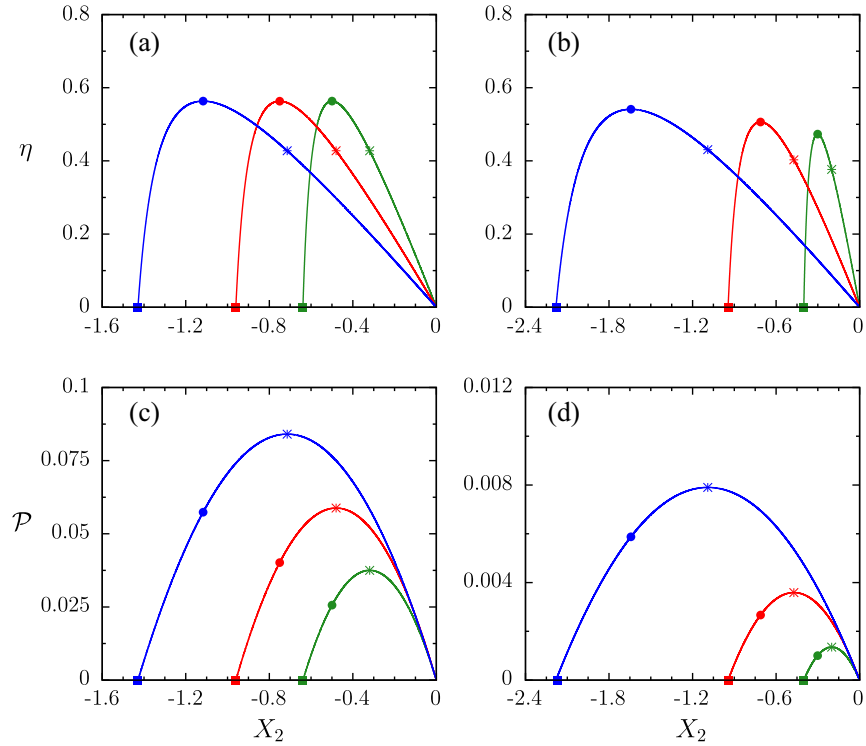

FIG. 2. Efficiency [(a) and (b)] and output power [(c) and (d)] (averaged over one period) for the isothermal work-to-work converter with $X_{1}=1, \tau=1, \gamma=1$, and different asymmetries $\kappa$ (from left to right, $\kappa=1.50,1.00$, and 0.67). (a) and (c) depict the results for constant drivings, whereas (b) and (d) depict those for linear drivings model. In all panels, squares, circles, and stars denote $X_{2 m S}$, $X_{2 M \eta}$, and $X_{2 M P}$, respectively.
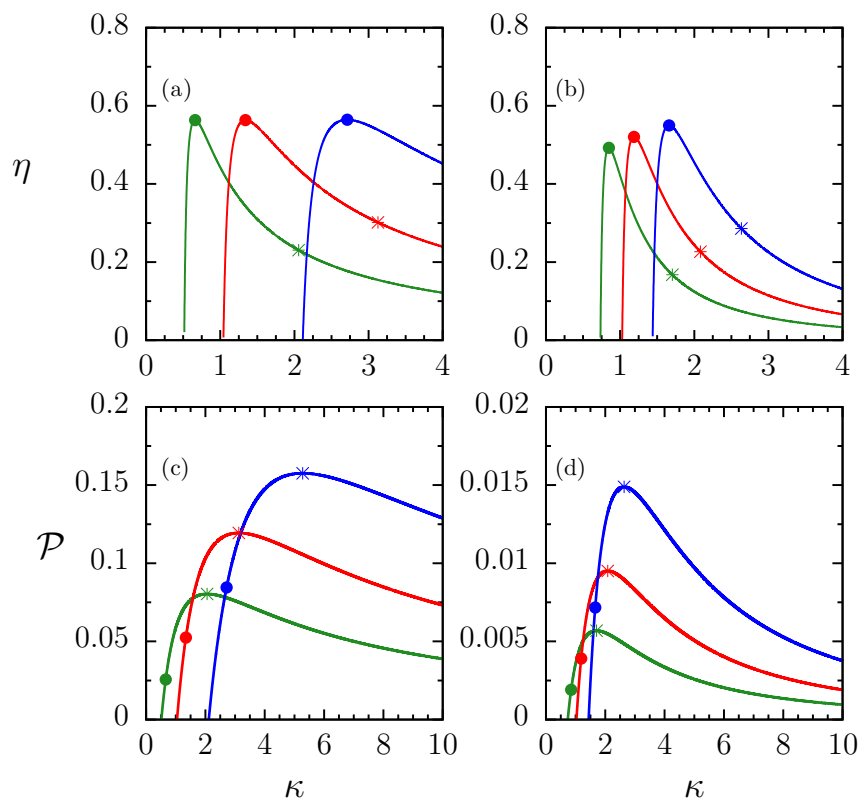

FIG. 3. Efficiency [(a) and (b)] and output power [(c) and (d)] (averaged over one period) for the isothermal work-to-work converter with $X_{1}=1, \tau=1, \gamma=1$, and different values of $X_{2}$ (from left to right, $X_{2}=-0.5,-1.0$, and -2.0 ). (a) and (c) depict the main results for the constant drivings model, while (b) and (d) depict those for the linear drivings model. In all panels, circles and stars denote $\kappa_{2 M \eta}$ and $\kappa_{2 M P}$, respectively. For such a set of parameters, the associated $\kappa_{2 m s}$ 's are out of the engine regime. 

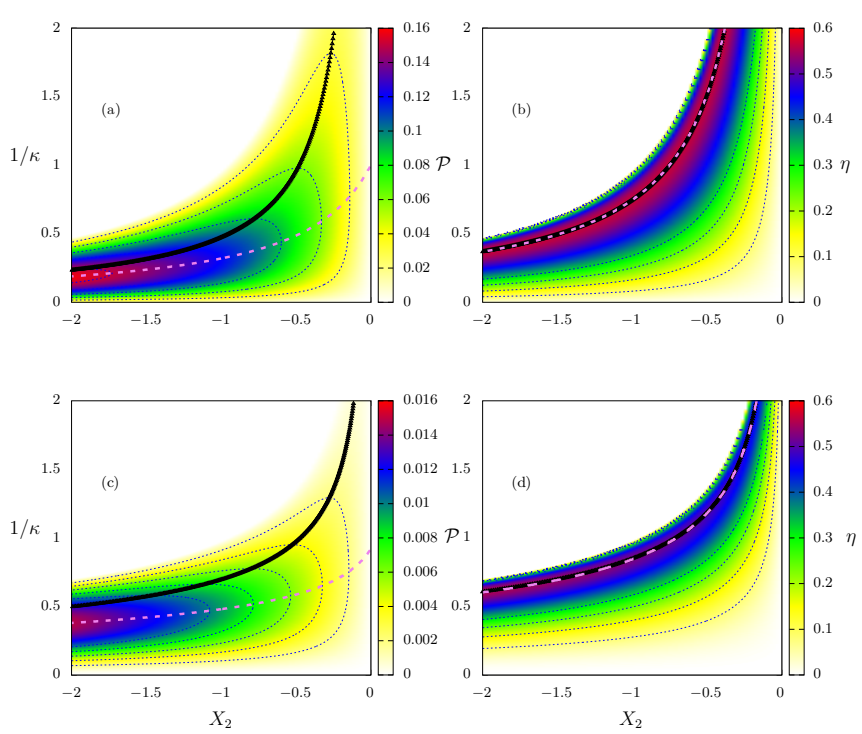

FIG. 4. For the isothermal work-to-work converter, the output power (left panels) and efficiency (right panels) for the constant [(a) and (b)] and linear [(c) and (d)] drivings models as a function of the inverse of the asymmetry parameter $\kappa$ and loading forces $X_{2}$. Dotted lines represent constant value loci; dashed and solid lines represent maximization with respect to $\kappa$ and $X_{2}$, respectively. $\mathrm{Pa}$ rameters: $\tau=1, \gamma=1, X_{1}=1$.

output for both force models as a function of the output force $X_{2}$ and asymmetry $\kappa$, respectively.

As discussed above, the engine regime operates for $X_{2 m}<$ $X_{2}<0$. An immediate advantage of the time asymmetry concerns the minimum output force $X_{2 m}$, which decreases with $\kappa$, implying that the engine regime interval increases with the asymmetry (see Fig. 2). Such a trend is consistent with the absorption of energy (average work rate $\overline{\dot{W}_{1}}$ ) for longer and longer times as $\kappa$ increases. Furthermore, the minimum entropy production (represented by the squares in Fig. 2) coincides with the minimum loading force (vanishing power output and efficiency) for constant drivings, but not for the linear case (although, for the values of the parameters used in Fig. 2, $X_{2 m S}$ is so close to $X_{m}$ that the difference is not discernible-it is of the order of $10^{-3}$ ).

The maximum efficiencies are almost constant for the constant force model [Fig. 2(a)] and slightly increase with $\kappa$ [Fig. 2(b)] for the linear force model. However, for small $\left|X_{2}\right|$, the efficiency is larger for the smaller values of $\kappa$. The effect of the time asymmetry on the output power is more pronounced. For both force models, the maximum power output clearly increases with $\kappa$.

Figure 4 depicts, for constant and linear drivings, a heat map for the power output and efficiency as a function of both the asymmetry and loading forces. For aesthetic reasons, they have been expressed in terms of $1 / \kappa($ instead of $\kappa)$ in the vertical axis. It is noteworthy that the maximum efficiency curves, represented by the dashed (solid) lines for the maximization with respect to $\kappa$ (loading force), are close to each other. Consequently, the choice of the parameter to maximize the efficiency is not important for either of the models presented here. Moreover, as previously discussed, the maximum effi- ciency is almost constant for the constant drivings model but increases with $\kappa$ for the linear drivings one. In contrast to the maximum efficiencies, the maximum power curves [Figs. 4(a) and 4(c) for constant and linear drivings, respectively] present rather different behaviors depending on the optimization parameter. The $\mathcal{P}_{M P, \kappa}$ curves (dashed lines) always lie below the $\mathcal{P}_{M P, X_{2}}$ (solid lines) ones, and they approach each other as $\kappa \rightarrow \infty$.

Finally, it is worth pointing out that while both drivings provide similar efficiencies, the constant driving case is clearly more advantageous than the linear one in terms of the output power.

\section{Simultaneous maximization of the asymmetry and the force}

One may also raise the relevant issue of maximizing the power output and efficiency with respect to the asymmetry and output force strength simultaneously. Although this is not possible in some cases (as explained below), we will proceed with presenting the framework assuming that such maximization is possible. As before, we shall restrict the analysis to drivings presenting a single physical solution for Eqs. (34) and (35). If this is not the case, each maximum of these equations should be analyzed individually to assert which is the global maximum in each case.

Under the assumption above, the maximum power output must satisfy simultaneously Eqs. (34) and (41), that is, we must find the optimal value of the asymmetry $\kappa_{M P}^{*}$ which satisfies the following condition:

$$
\frac{L_{21}^{\prime}\left(\kappa_{M P}^{*}\right)}{L_{22}^{\prime}\left(\kappa_{M P}^{*}\right)}=\frac{1}{2} \frac{L_{21}\left(\kappa_{M P}^{*}\right)}{L_{22}\left(\kappa_{M P}^{*}\right)} .
$$

Once the optimal asymmetry $\kappa_{M P}^{*}$ is obtained, the optimal force $X_{2 M P}^{*}$ is calculated from Eq. (41) and given by

$$
X_{2 M P}^{*}=-\frac{1}{2} \frac{L_{21}\left(\kappa_{M P}^{*}\right)}{L_{22}\left(\kappa_{M P}^{*}\right)} X_{1} .
$$

Graphically, the condition above is precisely the crossing point between lines for which the power (or efficiency) is maximized with respect to $X_{2}$ and $\kappa$. However, in some cases (as illustrated by the constant and linear drivings presented above) these two lines do not cross at all. The physical reason is that the power output keeps growing as $\kappa \rightarrow \infty$ (with an appropriate choice of value of $X_{2}$ for each $\kappa$ ). In other words, for such models, it is advantageous to apply a very large output force (in modulus) for a short period. Conversely, if the force model involves a rapidly decaying input driving $g_{1}(t)$ and growing output driving $g_{2}(t)$, an optimal output power may be found. In such a case, the power and efficiency at maximum power are readily evaluated as

$$
\mathcal{P}^{*}=\frac{k_{\mathrm{B}} T}{4} \frac{L_{21}^{2}\left(\kappa_{M P}^{*}\right)}{L_{22}\left(\kappa_{M P}^{*}\right)} X_{1}^{2}
$$

and

$$
\eta^{*}=\frac{L_{21}^{2}\left(\kappa_{M P}^{*}\right)}{4 L_{11}\left(\kappa_{M P}^{*}\right) L_{22}\left(\kappa_{M P}^{*}\right)-2 L_{21}\left(\kappa_{M P}^{*}\right) L_{12}\left(\kappa_{M P}^{*}\right)} .
$$

Thereby, the optimal output power increases quadratically with the input force while the efficiency is completely determined by the driving force model. It is noteworthy that, 

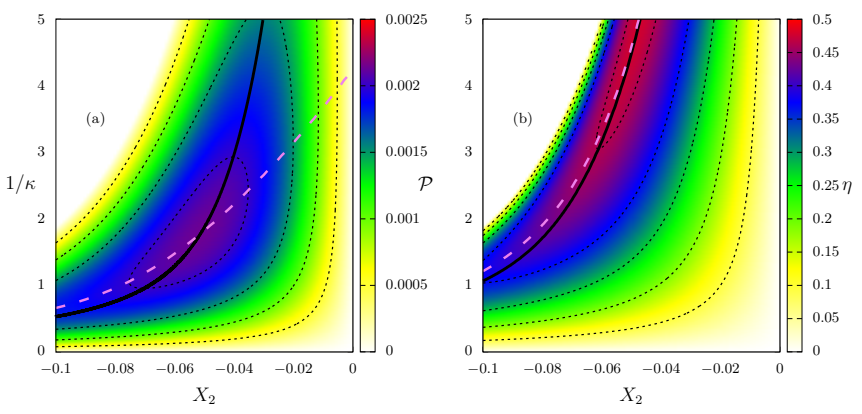

FIG. 5. For the exponential driving, depiction of output power (a) and efficiency (b) vs the inverse of the asymmetry coefficient $\kappa$ and the output force $X_{2}$ for $\tau=1, \gamma=1$, and $X_{1}=1$. Dotted lines represent constant value loci; dashed and solid lines represent maximization with respect to $\kappa$ and $X_{2}$, respectively.

despite the apparent temperature dependency of the power output in Eq. (52), the temperature cancels out when we use the expressions for the Onsager coefficients [see, e.g., Eq. (24)]. Similar expressions can be obtained for the simultaneous maximization of efficiency [by equaling the ratio $X_{2} / X_{1}$ from Eqs. (35) and (40)]. Since the expressions are more involved, we abstain from presenting them here. In order to illustrate the previous ideas, we consider an exponential driving given by

$$
\begin{gathered}
f_{1}(t)=X_{1} e^{-9 \gamma t}, \quad \text { for } 0 \leqslant t<\tau_{1}, \\
f_{2}(t)=X_{2} e^{\gamma\left(t-\tau_{1}\right)}, \quad \text { for } \tau_{1} \leqslant t<\tau .
\end{gathered}
$$

Figures 5(a) and 5(b) depict, for the above exponential drivings, the heat maps of the output power and efficiency as functions of $\kappa$ and $X_{2}$, respectively. Contrasting to the previous models, the crossing between maximum power curves is evident for the exponential drivings model above, and thereby the global optimization is possible. Although for the exponential model given by Eqs. (54) and (55) the crossing between maximum efficiency curves is absent, it does appear for other exponential driving choices [e.g., for $f_{1}(t)=X_{1} e^{-7 \gamma t}$ and $\left.f_{2}(t)=X_{2} e^{3 \gamma\left(t-\tau_{1}\right)}\right]$ and follow the theoretical prescription above.

\section{B. Thermal engine}

In this section, we derive general findings for thermal engines in which the particle is also exposed to distinct thermal baths in each stage. Although the power output $\mathcal{P}$ is the same as before (it does not depend on the temperatures), the efficiency may change because of the appearance of heat flow. Hence, in addition to the input energy received as work, the engine may also receive energy from the hot reservoir. Consequently, the maximization of power output with respect to the output force $X_{2 M P}$ or the asymmetry $\kappa_{M P}$ is the same as before, but the corresponding efficiencies may differ (if $\bar{Q}_{1}<0$ or $\bar{Q}_{2}<0$ ) from such a case, following Eq. (31) instead. Anyhow, the efficiency of the engine for reservoirs with different temperatures is always smaller than or equal to that for isothermal reservoirs.
From Eq. (11), the average heat dissipated by the Brownian particle per cycle while in contact with the $i$ reservoir $\bar{Q}_{i}$ can be obtained as

$$
\begin{aligned}
& \bar{Q}_{1}=\frac{m \gamma}{\tau}\left[\int_{0}^{\tau_{1}}\left\langle v_{1}\right\rangle^{2} d t-C\left(\tau_{1}\right)\left(\Gamma_{1}-\Gamma_{2}\right)\right], \\
& \bar{Q}_{2}=\frac{m \gamma}{\tau}\left[\int_{\tau_{1}}^{\tau}\left\langle v_{2}\right\rangle^{2} d t+C\left(\tau_{1}\right)\left(\Gamma_{1}-\Gamma_{2}\right)\right],
\end{aligned}
$$

where $C\left(\tau_{1}\right)=\operatorname{csch}(\gamma \tau) \sinh \left(\gamma \tau_{1}\right) \sinh \left(\gamma \tau_{2}\right) / 2 \gamma^{2}$ is strictly positive. Therefore, since the first term on the right-hand side of Eqs. (56) and (57) is positive, heat always flows into the colder reservoir. As with the hot reservoir, the heat may flow from or into the reservoir. For simplicity, we shall restrict our analysis to the case $\Gamma_{1}>\Gamma_{2}$, that is, the first reservoir being the hot one, but it is worth pointing out that all of the discussion below is valid for $\Gamma_{1}<\Gamma_{2}$ if we analyze Eq. (57) instead of Eq. (56).

For $\Gamma_{1}>\Gamma_{2}$, Eq. (56) ensures that heat flows into the system if $\int_{0}^{\tau_{1}}\left\langle v_{1}\right\rangle^{2} d t<C\left(\tau_{1}\right)\left(\Gamma_{1}-\Gamma_{2}\right)$. Physically, this condition is a balance between kinetic energy that flows into the system due to the forces and the dissipation. If $X_{1}$ is strong enough (or if the difference in temperature of the reservoirs is small enough), energy flows into both reservoirs. Thereby, the engine effectively reduces to an isothermal work-to-work converter, so that the efficiency is still described by Eq. (33) and all results and findings from Sec. III A regarding the efficiency optimization hold. Moreover, for small enough temperature differences, the engine efficiency is larger than the Carnot efficiency. This is possible because work-to-work conversion is not bounded by the thermodynamics laws the same way heat-to-work conversion is [43]. Otherwise, the inequality above is satisfied, and energy flows from the first reservoir into the engine. For $\Gamma_{1}<\Gamma_{2}$, the same energy balance occurs, but we need to assert the positiveness or negativeness of Eq. (57).

Furthermore, although exact, the achievement of general expressions for optimized efficiencies outside the isothermal work-to-work regime is more cumbersome than achieving the ones obtained for such a regime, making a general analysis unfeasible. Nevertheless, the discussion of a simple asymptotic limit is instructive. If the second term on the right-hand side of Eq. (56) [or Eq. (57)] is the dominant one, $\left|\Gamma_{1}-\Gamma_{2}\right| \gg 1$ and $\left|\bar{Q}_{1}\right| \gg\left|\bar{W}_{1}\right|$ (or $\left|\dot{Q}_{2}\right| \gg\left|\bar{W}_{1}\right|$ ). Therefore the efficiency becomes $\eta \approx-\mathcal{P} / \dot{Q}_{1}$ (or $\eta \approx-\mathcal{P} / \dot{Q}_{2}$ ), which maximization, with respect to $X_{2}$, yields $X_{2 M \eta} \approx X_{2 M P}$ and follows Eq. (41). Hence the corresponding $\eta_{M \eta}$ approaches to the following expression:

$$
\eta_{M \eta, X_{2}} \approx \frac{T_{2}}{8 \gamma^{2} T_{i} C\left(\tau_{1}\right)} \frac{L_{21}^{2}}{L_{22}} \tau X_{1}^{2} \ll 1,
$$

where $T_{i}$ is the temperature of the hot reservoir. When the hot bath is the first reservoir, the fact that the efficiency is small is directly observable since the factor $T_{2} / T_{1} \ll 1$. However, when the second reservoir is the hotter one, the temperature ratio becomes 1 , and the smallness of the efficiency comes from the Onsager coefficients: $L_{21}^{2} / L_{22} \propto 1 / T_{2}$. It is also worth mentioning that the apparent dependence on the period cancels out because the Onsager coefficients are proportional to $1 / \tau$ [see Eq. (24)]. Therefore, for high 

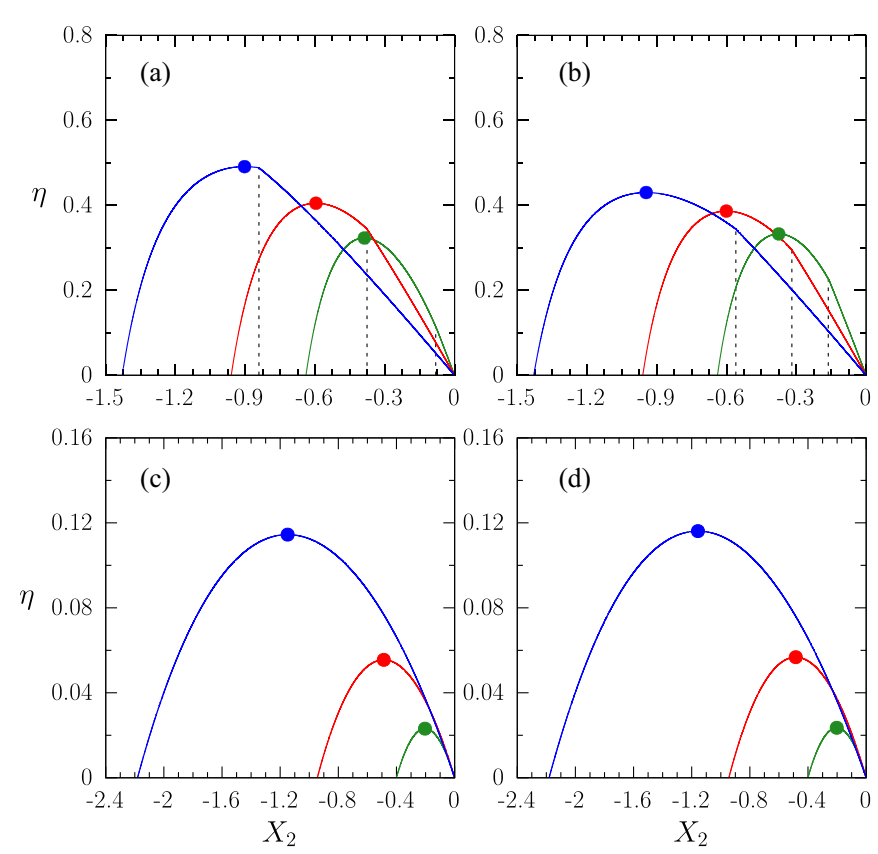

FIG. 6. Efficiency as a function of the force strength $X_{2}$ for the constant [(a) and (b)] and linear [(c) and (d)] drivings, respectively. Parameters: $\tau=1, \gamma=1$, and $X_{1}=1$ and distinct temperatures $\left[\Gamma_{1}=2.0\right.$ and $\Gamma_{2}=1.5$ in (a) and (c) and $\Gamma_{1}=1.5$ and $\Gamma_{2}=2.0$ in (b) and (d)]. Circles denote maximum efficiencies, and their $X_{2 M P}$ 's are the same as in Fig. 2. From left to right, $\kappa=1.50,1.00$, and 0.67). Dashed vertical lines stand for the values of $X_{2}$ for which $\bar{Q}_{i}$ changes sign ( $i$ being the index of the hot reservoir).

temperature differences, the engine efficiency is very small for any value of the asymmetry.

In order to illustrate our findings for reservoirs with different temperatures, we consider the constant and linear drivings models presented above. Figure 6 exemplifies, for distinct temperature reservoirs, the efficiency for the same values of $\kappa$ used in Fig. 2 for constant [Figs. 6(a) and 6(b)] and linear drivings [Figs. 6(c) and 6(d)], respectively. In Figs. 6(a) and 6 (c) the temperature of the first reservoir is larger than that of the second reservoir, while Figs. 6(b) and 6(d) depict the other way around.

In accordance with general findings from Sec. III B, for constant drivings there are two regimes (the vertical lines in Fig. 6 denote the values of $X_{2}$ which separate them) for which the heat exchanged between the Brownian particle and the hot reservoir changes sign. Conversely, they are not present for the linear drivings model [Figs. 6(c) and 6(d)] because the heat exchange with the hot reservoir does not change sign for the parameters used in the figures. Since $\left\langle v_{i}\right\rangle^{2}$ increases with $X_{2}^{2}$, the term coming from the difference of temperatures in Eq. (56) dominates over it when $\left|X_{2}\right|$ is small, and hence the machine is less efficient than the isothermal work-to-work converter. Conversely, for large $\left|X_{2}\right|$ the engine may become as efficient as the isothermal work-to-work converter if the exchanged heat with the hot reservoir changes sign [left of the lines in Figs. 6(a) and 6(b)]. Anyhow, by comparing the performance of the isothermal case with the differenttemperature case, we see that the decay in efficiency for linear drivings is more pronounced than that for constant drivings.

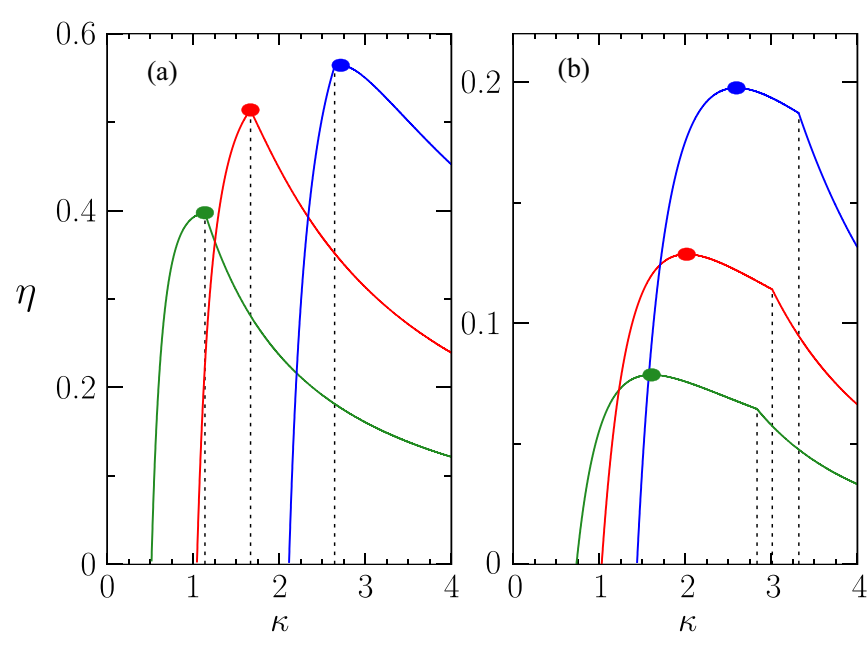

FIG. 7. Efficiency vs the time asymmetry $\kappa$ for the (a) constant and (b) linear drivings, respectively, for $\tau=1, \gamma=1, X_{1}=1$, and different temperatures $\left(\Gamma_{1}=2.0\right.$ and $\left.\Gamma_{2}=1.5\right)$. Circles denote maximum efficiencies, and their $X_{2 M P}$ 's are the same as in Fig. 3. From left to right, $X_{2}=-0.5,-1.0$, and -2.0 . Dashed vertical lines stand for the values of $\kappa$ for which $\bar{Q}_{1}$ changes sign. For such a set of parameters, $\kappa_{2 m s}$ are out of the engine regime.

As for isothermal reservoirs, the machine performance always improves as $\kappa$ increases, encompassing not only an extension of its operation regime $X_{2 m}$ but also a more pronounced increase in efficiencies, again, more substantial for linear drivings. Moreover, the asymmetry may be used to mitigate the drop in the efficiency produced by the different temperatures of the thermal reservoirs.

In Fig. 7, we show the efficiency as a function of the asymmetry for various values of $X_{2}$. Similarly to the previous figure, the vertical lines denote the values of $\kappa$ for which the heat from the hot reservoir changes sign and delimit the isothermal work-to-work converter regime. The discussion of whether the isothermal work-to-work converter regime lies to the left or right of the vertical lines is not so obvious because both $C\left(\tau_{1}\right)$ and $\left\langle v_{1}\right\rangle$ depend on the asymmetry. However, the work-to-work regime lies to the right of the lines, since the function $C\left(\tau_{1}\right)$ reaches its maximum for $\kappa=1\left(\tau_{1}=\tau / 2\right)$ and the first term on the right-hand side of Eq. (56) is expected to increase given that its limit of integration increases with $\kappa$.

Figure 8 presents heat maps of the efficiency for different temperature reservoirs as a function of the output force and asymmetry. By drawing a comparison with the isothermal work-to-work converter (Fig. 4), it reveals that the difference in temperature makes the choice of the optimization parameter (force strength or time asymmetry) more relevant. While both optimized lines lie almost on top of each other for the isothermal case, Fig. 8 shows that they are clearly distinct, particularly for the linear drivings. Another point to be addressed concerns that high efficiencies are restricted to larger $\left|X_{2}\right|$ 's for constant drivings when temperatures are different. This contrasts to its extension to smaller values for isothermal reservoirs [the hot (red) region in Fig. 4(b) has more spread than that in Fig. 8(a)]. Conversely, for linear drivings, the decrease in the efficiency extends for all values of $\kappa$ and $X_{2}$ when compared with the isothermal work-to-work converter 

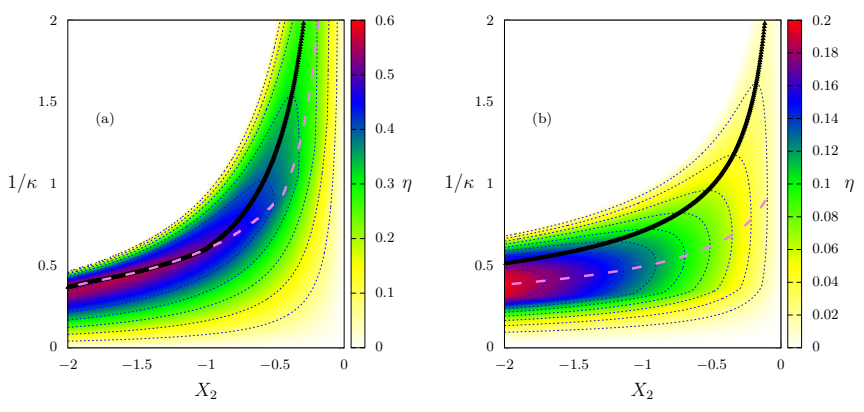

FIG. 8. Depiction of efficiency as a function of the inverse of the asymmetry coefficient $\kappa$ and the output force $X_{2}$, for (a) constant and (b) linear drivings, respectively. Solid and dashed lines denote the maximization with respect to $X_{2}$ and $\kappa$, respectively. Parameters: $\Gamma_{1}=2.0$ and $\Gamma_{2}=1.5, \tau=1, \gamma=1$, and $X_{1}=1$.

[note that efficiency in Fig. 4(d) is three times larger than that in Fig. 8(b)]. However, larger efficiencies in such a case are obtained solely for larger values of $\left|X_{2}\right|$ in a certain range of $\kappa$.

Lastly, we draw a comparison between the efficiency given by Eq. (31) with Eq. (45) from Ref. [33], which is based on the ratio between the entropy production fluxes. Although both expressions behave similarly and approach each other as $\Delta \Gamma \rightarrow 0$ (or $\Delta \Gamma \ll 1$ ), it is worth mentioning that the latter overestimates the efficiency as $\Delta \Gamma$ increases.

\section{CONCLUSIONS}

We introduced an alternative strategy for optimizing the performance of Brownian engines, based on the idea of asymmetric interaction time between the system (Brownian particle) and the thermal baths. Exact expressions for thermodynamic quantities and their maximized values were obtained, irrespective of the kind of driving and asymmetry. The time asymmetry can always be tuned to obtain a gain larger than in the symmetric case. In addition to the improvement of the power output and efficiency, the time asymmetry also enlarges the range of forces for which the system operates as an engine. Another advantage of asymmetric times is that they can be conveniently chosen for compensating part of the limitations due to the machine design, such as its operation period and the driving considered. Results for constant and linear drivings confirm that the appropriate tuning of the asymmetry produces gains for the efficiency substantially larger than those achieved for the symmetric case. It is important to point out that the symmetric engine does not necessarily operate inefficiently, as exemplified in Fig. 2. However, the tuning of the asymmetry, for given values of the other parameters (output force and period), provides a reliable route for enhancing the engine performance.

Contrariwise to usual machines, for which the heat flow due to the gradient of temperature is fundamental for the power extraction and enhancing the efficiency, in the present case the efficiency is higher for isothermal reservoirs. The reason for such behavior concerns that the energy exchange between the Brownian particle and the different thermal reservoirs occurs in different stages. Since the heat transfer and the output force are uncoupled, the heat flux cannot be converted into useful work. For instance, one would require drivings dependent on the velocity in order to be able to extract work from heat in the present model. Although the robustness of our findings has been verified for a few examples of drivings, our approach can be straightforwardly extended for other thermal machines, where in principle similar findings are expected. This is reinforced by recent results unveiling the importance of asymmetric times for optimizing the efficiency at maximum power of a quantum-dot thermal machine, which gain provides efficiencies larger than the Curzon-Ahlborn efficiency [44].

We finish this paper highlighting a couple of perspectives. While in this paper we analyzed the maximization of the output power and efficiency with respect to the time asymmetry and the output force strength, keeping the other parameters of the machine fixed, it might be worthwhile to study the maximization under different physical conditions, such as holding the dissipation or efficiency fixed. Finally, it might also be interesting to extend the role of asymmetric times for other kinds of drivings (e.g., velocity-dependent drivings providing extraction of useful work from heat) as well as for massive Brownian particles (underdamped case) in order to compare their performances.

\section{ACKNOWLEDGMENTS}

C.E.F. acknowledges the financial support from São Paulo Research Foundation (FAPESP) under Grants No. 2018/02405-1, No. 2021/05503-7, and No. 2021/03372-2. A.R. thanks Pronex/Fapesq-PB/CNPq (Grant No. 151/2018) and $\mathrm{CNPq}$ (Grant No. 308344/2018-9). The financial support from Coordenação de Aperfeiçoamento de Pessoal de Nível Superior, Brasil (CAPES) and CNPq are also acknowledged.

\section{APPENDIX A: ONSAGER COEFFICIENTS AND LINEAR REGIMES}

In this Appendix, we address the relation between coefficients $\tilde{L}_{i j}$ and Onsager coefficients $L_{i j}$. Our starting point is the steady-state entropy production averaged over one period, which is given by

$$
\bar{\Pi}=\frac{2 \gamma k_{\mathrm{B}}}{m}\left(\frac{\overline{\dot{Q}}_{1}}{\Gamma_{1}}+\frac{\overline{\dot{Q}}_{2}}{\Gamma_{2}}\right)=\bar{\Pi}_{F}+\bar{\Pi}_{T} .
$$

The coefficients $\tilde{L}_{i j}$ are straightforwardly obtained from $\bar{\Pi}_{F}$ performing the integration in Eq. (27) [in which the $\left\langle v_{i}\right\rangle(t)$ 's 
are given by Eq. (18)] as

$$
\begin{aligned}
\tilde{L}_{11} & =\frac{\gamma}{\tau}\left[\frac{\left(e^{2 \gamma\left(\tau-\tau_{1}\right)}-1\right) \hat{G}_{1}\left(\tau_{1}\right)^{2}}{\Gamma_{2}\left(e^{\gamma \tau}-1\right)^{2}}+\frac{\gamma}{\Gamma_{1}} \int_{0}^{\tau_{1}} \frac{2 e^{-2 \gamma t}\left[\left(e^{\gamma \tau}-1\right) \hat{G}_{1}(t)+\hat{G}_{1}\left(\tau_{1}\right)\right]^{2}}{\left(e^{\gamma \tau}-1\right)^{2}} d t\right], \\
\tilde{L}_{22} & =\frac{\gamma}{\tau}\left[\frac{\left(1-e^{-2 \gamma \tau_{1}}\right) \hat{G}_{2}(\tau)^{2}}{\Gamma_{1}\left(e^{\gamma \tau}-1\right)^{2}}+\frac{\gamma}{\Gamma_{2}} \int_{\tau_{1}}^{\tau} \frac{2 e^{-2 \gamma t}\left[\left(e^{\gamma \tau}-1\right) \hat{G}_{2}(t)+\hat{G}_{2}(\tau)\right]^{2}}{\left(e^{\gamma \tau}-1\right)^{2}} d t\right], \\
\tilde{L}_{12}+\tilde{L}_{21} & =\frac{2 \gamma e^{-\gamma \tau_{1}} \hat{G}_{1}\left(\tau_{1}\right) \hat{G}_{2}(\tau)}{\tau\left(e^{\gamma \tau}-1\right)^{2}}\left[\frac{\sinh \left(\gamma \tau_{1}\right)}{\Gamma_{1}}+\frac{\sinh \left(\gamma\left(\tau-\tau_{1}\right)\right)}{\Gamma_{2}}\right] \\
& +\frac{2 \gamma^{2}}{\Gamma_{1} \Gamma_{2} \tau\left(e^{\gamma \tau}-1\right)}\left[\Gamma_{2} \hat{G}_{2}(\tau) \int_{0}^{\tau_{1}} \hat{G}_{1}(t) e^{-2 \gamma t} d t+\Gamma_{1} \hat{G}_{1}\left(\tau_{1}\right) \int_{\tau_{1}}^{\tau} \hat{G}_{2}(t) e^{\gamma(\tau-2 t)} d t\right],
\end{aligned}
$$

where $\hat{G}_{i}(t)=\int_{\tau_{i-1}}^{t} g_{i}\left(t^{\prime}\right) d t^{\prime}$. For equal temperatures $\Gamma_{1}=$ $\Gamma_{2}=\Gamma, \bar{\Pi}$ reduces to the following expression:

$$
\begin{aligned}
\bar{\Pi} & =\bar{\Pi}_{F}=-\frac{2 \gamma k_{\mathrm{B}}}{m \Gamma}\left(\overline{\dot{W}}_{1}+\overline{\dot{W}}_{2}\right) \\
& =L_{11} X_{1}^{2}+\left(L_{12}+L_{21}\right) X_{1} X_{2}+L_{22} X_{2}^{2} .
\end{aligned}
$$

Hence, for isothermal reservoirs, the entropy production can be written in terms of the Onsager coefficients even in the nonlinear (force) regime and thereby $\tilde{L}_{i j}=L_{i j}$. Conversely, for the thermal linear regime, it is convenient to express $\Gamma_{1}$ and $\Gamma_{2}$ in terms of the difference of temperatures $\Gamma_{1}=\Gamma-$ $\Delta \Gamma$ and $\Gamma_{2}=\Gamma+\Delta \Gamma$. In such a case, Eq. (A1) becomes

$$
\bar{\Pi} \approx \frac{2 \gamma k_{\mathrm{B}}}{m \Gamma}\left[-\left(\overline{\dot{W}}_{1}+\overline{\dot{W}}_{2}\right)+\left(\overline{\dot{Q}}_{1}-\overline{\dot{Q}}_{2}\right) \frac{\Delta \Gamma}{\Gamma}\right] .
$$

Let us assume that $\tilde{L}_{i j}$ can be expanded in a power series of the temperature difference, $\tilde{L}_{i j}=L_{i j}^{(0)}+L_{i j}^{(c)} \Delta \Gamma$, where $L_{i j}^{(0)}$ is the coefficient for $\Gamma_{1}=\Gamma_{2}=\Gamma$ and $L_{i j}^{(c)}$ is the first-order correction. In terms of such coefficients, the average entropy production $\bar{\Pi}$ is given by

$$
\begin{aligned}
\bar{\Pi} & =\bar{\Pi}_{F}+\bar{\Pi}_{T} \\
& =\left[L_{11}^{(0)} X_{1}^{2}+\left(L_{12}^{(0)}+L_{21}^{(0)}\right) X_{1} X_{2}+L_{22}^{(0)} X_{2}^{2}\right] \\
& +\left[L_{11}^{(c)} X_{1}^{2}+\left(L_{12}^{(c)}+L_{21}^{(c)}\right) X_{1} X_{2}+L_{22}^{(c)} X_{2}^{2}\right] \Delta \Gamma \\
& +\frac{4 L_{\Gamma \Gamma}}{\Gamma^{2}}(\Delta \Gamma)^{2} .
\end{aligned}
$$

By comparing Eqs. (A4) and (A5), it follows that

$L_{11}^{(0)} X_{1}^{2}+\left(L_{12}^{(0)}+L_{21}^{(0)}\right) X_{1} X_{2}+L_{22}^{(0)} X_{2}^{2}=-\frac{2 \gamma k_{\mathrm{B}}}{m \Gamma}\left(\overline{\dot{W}}_{1}+\overline{\dot{W}}_{2}\right)$,

and hence Onsager coefficients $L_{i j}$ correspond to zeroth-order coefficients $L_{i j}^{(0)}$ evaluated from $\bar{\Pi}_{F}$. Once again, they do not depend on $\Delta \Gamma$, since $\bar{W}_{i}$ does not depend on the temperature at all.

In the true linear regime (both temperature gradient and force strength are small), the correction of $\bar{\Pi}_{F}$ is of third order $\left(X_{i} X_{j} \Delta \Gamma\right)$; thus it can be neglected. Hence the entropy production components $\bar{\Pi}_{F}$ and $\bar{\Pi}_{T}$ are approximately

$$
\bar{\Pi}_{F} \approx-\frac{2 \gamma k_{\mathrm{B}}}{m \Gamma}\left(\bar{W}_{1}+\overline{\dot{W}}_{2}\right)
$$

and

$$
\bar{\Pi}_{T} \approx \frac{4 L_{\Gamma \Gamma}}{\Gamma^{2}}(\Delta \Gamma)^{2},
$$

respectively. In addition, the coefficients $\tilde{L}_{i j}$ and $L_{i j}$ are approximately equal, $\tilde{L}_{i j} \approx L_{i j}$.

\section{APPENDIX B: CONSTANT DRIVINGS}

For the machine operating at constant drivings, defined by the forces from Eqs. (46) and (47), the velocities $\left\langle v_{i}\right\rangle(t)$ are given by

$$
\begin{array}{r}
\left\langle v_{1}\right\rangle(t)=\frac{X_{1}}{\gamma}+\frac{e^{-\gamma\left(t-\tau_{1}\right)}-e^{-\gamma(t-\tau)}}{e^{\gamma \tau}-1} \frac{X_{1}-X_{2}}{\gamma}, \\
\left\langle v_{2}\right\rangle(t)=\frac{X_{2}}{\gamma}+\frac{e^{-\gamma\left(t-\tau-\tau_{1}\right)}-e^{-\gamma(t-\tau)}}{e^{\gamma \tau}-1} \frac{X_{1}-X_{2}}{\gamma},
\end{array}
$$

for $i=1$ and 2, respectively. The associated Onsager coefficients are straightforwardly obtained from Eq. (24) and are given by

$$
\begin{aligned}
& L_{11}=\frac{2 \tau_{1}}{\Gamma_{1} \tau}-L_{12}, \\
& L_{22}=\frac{2 \tau_{2}}{\Gamma_{2} \tau}-L_{21}, \\
& L_{12}=\frac{4 \operatorname{csch}\left(\frac{\gamma \tau}{2}\right) \sinh \left(\frac{\gamma \tau_{1}}{2}\right) \sinh \left(\frac{1}{2} \gamma \tau_{2}\right)}{\gamma \Gamma_{1} \tau}, \\
& L_{21}=\frac{4 \operatorname{csch}\left(\frac{\gamma \tau}{2}\right) \sinh \left(\frac{\gamma \tau_{1}}{2}\right) \sinh \left(\frac{1}{2} \gamma \tau_{2}\right)}{\gamma \Gamma_{2} \tau} .
\end{aligned}
$$

Furthermore, for isothermal reservoirs, $L_{12}$ and $L_{21}$ are equal for any value of asymmetry parameter $\kappa=\tau_{1} / \tau_{2}$.

\section{APPENDIX C: LINEAR DRIVINGS}

Similarly to the constant drivings model, the average velocities for the linear driving model [defined by Eqs. (48) and 
(49)] are obtained from Eq. (18) and are given by

$$
\left\langle v_{1}\right\rangle(t)=\frac{1}{\gamma}\left\{X_{1}(\gamma t-1)+\frac{e^{-\gamma t}}{e^{\gamma \tau}-1}\left\{X_{1}\left[e^{\gamma \tau}+e^{\frac{\gamma \kappa \tau}{1+\kappa}}\left(\frac{\gamma \kappa \tau}{1+\kappa}-1\right)\right]-X_{2}\left[e^{\frac{\gamma \kappa \tau}{1+\kappa}}+e^{\gamma \tau}\left(\frac{\gamma \tau}{1+\kappa}-1\right)\right]\right\}\right\}
$$

and

$$
\left\langle v_{2}\right\rangle(t)=\frac{1}{\gamma}\left\{X_{2}\left[1-\gamma\left(t-\frac{\kappa \tau}{1+\kappa}\right)\right]+\frac{e^{-\gamma\left(t-\frac{\kappa \tau}{1+\kappa}\right)}}{e^{\gamma \tau}-1}\left\{X_{1}\left[e^{\frac{\gamma \tau}{1+\kappa}}+e^{\gamma \tau}\left(\frac{\gamma \kappa \tau}{1+\kappa}-1\right)\right]-X_{2}\left[e^{\frac{\gamma \tau}{1+\kappa}}\left(\frac{\gamma \tau}{1+\kappa}-1\right)+e^{\gamma \tau}\right]\right\}\right\} .
$$

Likewise, Onsager coefficients $L_{i j}$ are also straightforwardly calculated from Eq. (24) and read

$$
\begin{aligned}
& L_{11}=\frac{2 \gamma^{3} \tau_{1}^{3}+\left[6-3 \gamma^{2} \tau_{1}^{2}\right] \operatorname{coth}\left(\frac{\gamma \tau}{2}\right)+6 \operatorname{csch}\left(\frac{\gamma \tau}{2}\right)\left[\gamma \tau_{1} \sinh \left(\frac{\gamma\left(\tau_{1}-\tau_{2}\right)}{2}\right)-\cosh \left(\frac{\gamma\left(\tau_{1}-\tau_{2}\right)}{2}\right)\right]}{3 \gamma \Gamma \tau}, \\
& L_{22}=\frac{2 \gamma^{3} \tau_{2}^{3}+\left[6-3 \gamma^{2} \tau_{2}^{2}-6 \cosh \left(\gamma \tau_{1}\right)\right] \operatorname{coth}\left(\frac{\gamma \tau}{2}\right)+6 \gamma \tau_{2} \operatorname{csch}\left(\frac{\gamma \tau}{2}\right) \sinh \left(\frac{\gamma\left(\tau_{2}-\tau_{1}\right)}{2}\right)+6 \sinh \left(\gamma \tau_{1}\right)}{3 \gamma \Gamma \tau}, \\
& L_{12}=\frac{2}{\gamma \tau \Gamma_{1}\left(1-e^{\gamma \tau}\right)}\left[1+\gamma \tau_{1}-e^{\gamma \tau_{1}}\right]\left[1-e^{\gamma \tau_{2}}\left(1-\gamma \tau_{2}\right)\right], \\
& L_{21}=\frac{2}{\gamma \tau \Gamma_{2}\left(1-e^{\gamma \tau}\right)}\left[1+\gamma \tau_{2}-e^{\gamma \tau_{2}}\right]\left[1-e^{\gamma \tau_{1}}\left(1-\gamma \tau_{1}\right)\right] .
\end{aligned}
$$

Notably, contrasting to the constant drivings case, coefficients $L_{12}$ and $L_{21}$ are different from each other when $\Gamma_{1}=\Gamma_{2}$. Only for symmetric switching times $\left(\tau_{1}=\tau_{2}\right)$ does it turn out that $L_{12}=L_{21}$.

[1] H. B. Callen, Thermodynamics and an Introduction to Thermostatistics (Wiley, New York, 1998).

[2] I. Prigogine, Introduction to Thermodynamics of Irreversible Processes (Interscience, New York, 1965).

[3] S. R. De Groot and P. Mazur, On the Theory of the Refractive Index of Non-polar Gases. II. Statistical Part (North-Holland, Amsterdam, 1962).

[4] U. Seifert, Stochastic thermodynamics, fluctuation theorems and molecular machines, Rep. Prog. Phys. 75, 126001 (2012).

[5] C. Van den Broeck, Thermodynamic Efficiency at Maximum Power, Phys. Rev. Lett. 95, 190602 (2005).

[6] G. Verley, M. Esposito, T. Willaert, and C. Van den Broeck, The unlikely Carnot efficiency, Nat. Commun. 5, 4721 (2014).

[7] T. Schmiedl and U. Seifert, Efficiency at maximum power: An analytically solvable model for stochastic heat engines, EPL (Europhys. Lett.) 81, 20003 (2007).

[8] M. Esposito, K. Lindenberg, and C. Van den Broeck, Universality of Efficiency at Maximum Power, Phys. Rev. Lett. 102, 130602 (2009).

[9] B. Cleuren, B. Rutten, and C. Van den Broeck, Universality of efficiency at maximum power, Eur. Phys. J.: Spec. Top. 224, 879 (2015).

[10] M. Esposito, R. Kawai, K. Lindenberg, and C. Van den Broeck, Quantum-dot Carnot engine at maximum power, Phys. Rev. E 81, 041106 (2010).

[11] U. Seifert, Efficiency of Autonomous Soft Nanomachines at Maximum Power, Phys. Rev. Lett. 106, 020601 (2011).

[12] Y. Izumida and K. Okuda, Efficiency at maximum power of minimally nonlinear irreversible heat engines, EPL (Europhys. Lett.) 97, 10004 (2012).

[13] N. Golubeva and A. Imparato, Efficiency at Maximum Power of Interacting Molecular Machines, Phys. Rev. Lett. 109, 190602 (2012).
[14] V. Holubec, An exactly solvable model of a stochastic heat engine: optimization of power, power fluctuations and efficiency, J. Stat. Mech.: Theory Exp. (2014) P05022.

[15] M. Bauer, K. Brandner, and U. Seifert, Optimal performance of periodically driven, stochastic heat engines under limited control, Phys. Rev. E 93, 042112 (2016).

[16] K. Proesmans, B. Cleuren, and C. Van den Broeck, PowerEfficiency-Dissipation Relations in Linear Thermodynamics, Phys. Rev. Lett. 116, 220601 (2016).

[17] Z. C. Tu, Efficiency at maximum power of Feynman's ratchet as a heat engine, J. Phys. A: Math. Theor. 41, 312003 (2008).

[18] S. Ciliberto, Experiments in Stochastic Thermodynamics: Short History and Perspectives, Phys. Rev. X 7, 021051 (2017).

[19] M. V. S. Bonança, Approaching Carnot efficiency at maximum power in linear response regime, J. Stat. Mech.: Theory Exp. (2019) 123203.

[20] B. Rutten, M. Esposito, and B. Cleuren, Reaching optimal efficiencies using nanosized photoelectric devices, Phys. Rev. B 80, 235122 (2009).

[21] K. Proesmans and C. Van den Broeck, The underdamped Brownian duet and stochastic linear irreversible thermodynamics, Chaos 27, 104601 (2017).

[22] I. A. Martínez, É. Roldán, L. Dinis, D. Petrov, J. M. R. Parrondo, and R. A. Rica, Brownian Carnot engine, Nat. Phys. 12, 67 (2016).

[23] K. Proesmans, Y. Dreher, M. Gavrilov, J. Bechhoefer, and C. Van den Broeck, Brownian Duet: A Novel Tale of Thermodynamic Efficiency, Phys. Rev. X 6, 041010 (2016).

[24] S. Krishnamurthy, S. Ghosh, D. Chatterji, R. Ganapathy, and A. K. Sood, A micrometre-sized heat engine operating between bacterial reservoirs, Nat. Phys. 12, 1134 (2016).

[25] V. Blickle and C. Bechinger, Realization of a micrometre-sized stochastic heat engine, Nat. Phys. 8, 143 (2012). 
[26] P. A. Quinto-Su, A microscopic steam engine implemented in an optical tweezer, Nat. Commun. 5, 5889 (2014).

[27] A. Kumar and J. Bechhoefer, Nanoscale virtual potentials using optical tweezers, Appl. Phys. Lett. 113, 183702 (2018).

[28] M. Esposito, R. Kawai, K. Lindenberg, and C. Van den Broeck, Efficiency at Maximum Power of Low-Dissipation Carnot Engines, Phys. Rev. Lett. 105, 150603 (2010).

[29] T. Schmiedl and U. Seifert, Optimal Finite-Time Processes in Stochastic Thermodynamics, Phys. Rev. Lett. 98, 108301 (2007).

[30] N. Pancotti, M. Scandi, M. T. Mitchison, and M. PerarnauLlobet, Speed-ups to Isothermality: Enhanced Quantum Thermal Machines through Control of the System-Bath Coupling, Phys. Rev. X 10, 031015 (2020).

[31] N. Piccione, G. De Chiara, and B. Bellomo, Power maximization of two-stroke quantum thermal machines, Phys. Rev. A 103, 032211 (2021).

[32] P. Abiuso and M. Perarnau-Llobet, Optimal Cycles for LowDissipation Heat Engines, Phys. Rev. Lett. 124, 110606 (2020).

[33] A. L. L. Stable, C. E. F. Noa, W. G. C. Oropesa, and C. E. Fiore, Thermodynamics of collisional models for Brownian particles: General properties and efficiency, Phys. Rev. Research 2, 043016 (2020).

[34] C. H. Bennett, The thermodynamics of computation-a review, Int. J. Theor. Phys. 21, 905 (1982).
[35] K. Maruyama, F. Nori, and V. Vedral, Colloquium: The physics of Maxwell's demon and information, Rev. Mod. Phys. 81, 1 (2009).

[36] T. Sagawa, Thermodynamic and logical reversibilities revisited, J. Stat. Mech.: Theory Exp. (2014) P03025.

[37] J. M. R. Parrondo, J. M. Horowitz, and T. Sagawa, Thermodynamics of information, Nat. Phys. 11, 131 (2015).

[38] T. Tomé and M. J. De Oliveira, Stochastic Dynamics and Irreversibility (Springer, Cham, Switzerland, 2015).

[39] T. Tomé and M. J. de Oliveira, Entropy production in irreversible systems described by a Fokker-Planck equation, Phys. Rev. E 82, 021120 (2010).

[40] T. Tomé and M. J. de Oliveira, Stochastic approach to equilibrium and nonequilibrium thermodynamics, Phys. Rev. E 91, 042140 (2015).

[41] S. Liepelt and R. Lipowsky, Operation modes of the molecular motor kinesin, Phys. Rev. E 79, 011917 (2009).

[42] B. Altaner, A. Wachtel, and J. Vollmer, Fluctuating currents in stochastic thermodynamics. II. Energy conversion and nonequilibrium response in kinesin models, Phys. Rev. E 92, 042133 (2015).

[43] J. M. Horowitz and M. Esposito, Work producing reservoirs: Stochastic thermodynamics with generalized Gibbs ensembles, Phys. Rev. E 94, 020102(R) (2016).

[44] P. E. Harunari, F. S. Filho, C. E. Fiore, and A. Rosas, Maximal power for heat engines: Role of asymmetric interaction times, Phys. Rev. Research 3, 023194 (2021). 